\title{
NUMERICAL MODELING OF THE PROCESS TEMPERATURE DISTRIBUTION AND ITS SOLUTION IN THE MATHCAD SYSTEM Allamuratov Sh.Z. ${ }^{1}$, Nurimov P.B. ${ }^{2}$, Kuvandikova D.K. ${ }^{3}$, Ziuatdinov I.Sh. ${ }^{4}$ (Republic of Uzbekistan) Email: Allamuratov341@scientifictext.ru
}

\author{
${ }^{I}$ Allamuratov Sharapatdin Ziuatdinovich - Candidate of Physical and Mathematical Sciences, Senior Lecturer, \\ DEPARTMENT OF INFORMATION EDUCATIONAL TECHNOLOGIES; \\ ${ }^{2}$ Nurimov Parakhat Baymuratovich - Teacher, \\ DEPARTMENT OF INFORMATION TECHNOLOGIES; \\ ${ }^{3}$ Kuvandikova Damegul Kazakbaevna - Teacher, \\ DEPARTMENT OF NATURAL AND GENERAL PROFESSIONAL SUBJECTS \\ ${ }^{4}$ Ziuatdinov Islambek Sharapatdinovich - Bachelor Student, \\ NUKUS BRANCH \\ TASHKENT UNIVERSITY OF INFORMATION TECHNOLOGY NAMED AFTER MUHAMMAD AL-KHORAZMI, \\ NUKUS, REPUBLIC OF UZBEKISTAN
}

\begin{abstract}
L. Where the boundary conditions are given in the following form: at the point $x=0$ we have the temperature, and at the point $x=L$ we have the temperature. And the initial time $t=0$, the propagation of temperature along the length of the rod is given in the form. This mathematical model (1) is replaced by difference equations in the form (3). The boundary-value problem is solved by the sweep method, using explicit schemes. The program is compiled on MathCad where the stability conditions for explicit schemes are performed using the formula $\lambda=\frac{k}{h^{2}}<0,5$.

Keywords: difference equation, sweep method, explicit scheme, stability.

\section{ЧИСЛЕННОЕ МОДЕЛИРОВАНИЕ ПРОЦЕССА РАСПРОСТРАНЕНИЯ ТЕМПЕРАТУРЫ И ЕГО РЕШЕНИЕ В СИСТЕМЕ МАТНСАD} Алламуратов Ш.3. ${ }^{1}$, Нуримов П.Б. ${ }^{2}$, Кувандикова Д.К. ${ }^{3}$, Зиуатдинов И.Ш. ${ }^{4}$ (Республика Узбекистан)

\footnotetext{
${ }^{1}$ Алламуратов Шарапатдин Зиуатдинович - кандидат физико-математических наук, стариий преподаватель, кафедра информачионных образовательных технологий;

${ }^{2}$ Нуримов Парахат Баймуратович - преподаватель, кафедра информачионных технологий;

${ }^{3}$ Кувандикова Дамегул Казакбаевна - преподаватель, кафедра естественных и общих профессиональных предметов;

${ }^{4}$ Зиатдинов Исламбек Шарапатдинович - студент бакалавриата, Нукусский филиал

Ташкентский университет информаџионных технологий им. Мухаммада аль-Хоразми, г. Нукус, Республика Узбекистан
}

Аннотация: в данной работе рассматривается задача распространения температуры по металлическому стержню длиной $L$, где граничные условия заданы в следующем виде: в точке $x=0$ имеем $u(0, t)=f_{0}(t)$ температуру, а в точке $x=L$ имеем $u(L, t)=f_{1}(t)$ температуру. A начальное время $t=0$, распространения температуры по длине стержня задано в виде $u(x, 0)=g(x)$. Данная математическая модель (1) заменена разностными уравнениями в виде (3). Краевая задача решена методом прогонки, с использованием явных схем. Программа составлена на MathCade, где условия устойчивости для явных схем выполняются по формуле $\lambda=\frac{k}{h^{2}}<0,5$. Ключевые слова: разностное уравнение, метод прогонки, явная схема, устойчивость.

В данной работе рассматривается задача распространения температуры по металлическому стержню длиной $0 \leq \mathrm{x} \leq \mathrm{L}$. Пусть в точке $\mathrm{x}=0$ имеем $u(0, t)=f_{0}(t)$ температуру, а в точке $\mathrm{x}=\mathrm{L}$ имеем $u(L, t)=f_{1}(t)$ температуру. Пусть в начальное время $\mathrm{t}=0$, распространения температуры по длине 
стержня зададим $u(x, 0)=g(x)$. Тогда функция распространения температуры по всему времени и по длине стержню определяется функцией $u(x, t)$ и задаётся следующим уравнением [1].

$$
\begin{aligned}
& u_{x x}=a u_{t} \\
& 0<x<L, \quad t>0
\end{aligned}
$$

где $a=\frac{c \rho}{k}$, с- температурная ёмкость материала стержня, $\rho$ - плотность материала, $\mathrm{k}$ температура проводимость. Сделаем замену переменных по времени чтобы $a=1$. Тогда математическая модель распространения температуры задаётся следующим уравнением в начальных и граничных условиях [2].

$$
\begin{aligned}
& u_{x x}=u_{t} \\
& u(x, 0)=g(x), \\
& u(0, t)=f_{1}(t), \\
& u(L, t)=f_{2}(t),
\end{aligned}
$$

где $0 \leq x \leq L, \quad 0 \leq t \leq T$.

Это называется уравнением диффузии илиуравнением теплопроводности. Зададим сеточную уравнению следующей разностной схемой.

$$
u_{x}=\frac{u_{i+1, j}-u_{i, j}}{h}, \quad u_{x x}=\frac{u_{i-1, j}-2 u_{i, j}+u_{i+1, j}}{h^{2}}, u_{t}=\frac{u_{i, j+1}-u_{i, j}}{k} .
$$

Имеем следующее разностнее уравнение [2].

$$
\frac{u_{i-1, j}-2 u_{i, j}+u_{i+1, j}}{h^{2}}=\frac{u_{i, j+1}-u_{i, j}}{k} .
$$

Начальные и граничные условия

$$
\begin{aligned}
& u_{i, j}=\lambda u_{i+1, j}+\lambda u_{i-1, j}+(1-2 \lambda) u_{i, j} \\
& u_{i, 0}=g(i h), \quad u_{0, j}=f_{1}(j k), \quad u_{n, j}=f_{2}(j k) .
\end{aligned}
$$

Условия устойчивости для явных схем $\lambda=\frac{k}{h^{2}}<0,5$.

Приведем пример для уравнения теплопроводности следующим образом.

$$
\begin{aligned}
& u_{x x}=u_{t} \\
& u(x, 0)=g(x), \\
& u(0, t)=g\left(x_{0}\right), \\
& u(L, t)=g\left(x_{n}\right) . \\
& \text { где } \quad 0 \leq x \leq L, \quad 0 \leq t \leq T .
\end{aligned}
$$

Если начальную температуру задать в виде функции, $g(x)=\left(\frac{1}{e^{5 x}}\right)+\tan (x)$ то определим распространение температуры по всему стержню.

Разностное уравнение в начальном и граничных условиях имеет следующий вид. 


$$
\begin{aligned}
& u_{i, j}=\lambda u_{i+1, j}+\lambda u_{i-1, j}+(1-2 \lambda) u_{i, j}, \\
& u_{i, 0}=g\left(x_{0}+i h\right), \\
& u_{i, 0}=g\left(x_{0}\right), \\
& u_{n, j}=g\left(x_{n}\right) .
\end{aligned}
$$

Здесь выполняются условия устойчивости $\lambda=\frac{k}{h^{2}}=0,417<0,5$.

Результаты вычислены в пакете MathCad. Из рисунков видно что $T_{i, 0}$ - распространение температуры

\begin{tabular}{|c|c|c|c|c|c|c|c|c|c|c|c|}
\hline & 0 & 1 & 2 & 3 & 4 & 5 & 6 & 7 & 8 & 9 & 10 \\
\hline 0 & 1 & 1 & 1 & 1 & 1 & 1 & 1 & 1 & 1 & 1 & 1 \\
\hline 1 & 0.707 & .772 & 0.8 & 0.824 & 0.841 & 0.855 & 0.867 & 0.878 & 0.888 & .896 & 0.904 \\
\hline 2 & 0.571 & 0.611 & 0.657 & 0.688 & 0.716 & 0.74 & 0.761 & 0.779 & 0.796 & 0.812 & 0.826 \\
\hline 3 & 0.532 & 0.559 & 0.588 & 0.62 & 0.648 & 0.675 & 0.698 & 0.721 & 0.742 & 0.761 & 0.78 \\
\hline 4 & 0.558 & 0.577 & 0.597 & 0.619 & 0.643 & 0.667 & 0.69 & 0.713 & 0.735 & 0.755 & 0.775 \\
\hline 5 & 0.628 & 0.643 & 0.659 & 0.676 & 0.695 & 0.716 & 0.736 & 0.757 & 0.777 & 0.797 & 0.816 \\
\hline 6 & 0.734 & 0.748 & 0.763 & 0.779 & 0.796 & 0.813 & 0.832 & 0.85 & 0.868 & 0.885 & 0.902 \\
\hline 7 & 0.872 & 0.888 & 0.904 & 0.922 & 0.939 & 0.955 & 0.97 & 0.986 & 1 & 1.015 & 1.029 \\
\hline 8 & 1.048 & 1.068 & 1.09 & 1.106 & 1.121 & 1.133 & 1.146 & 1.157 & 1.167 & 1.178 & 1.188 \\
\hline \begin{tabular}{|l|}
9 \\
\end{tabular} & 1.271 & 1.3 & 1.313 & 1.325 & 1.333 & 1.341 & 1.347 & 1.354 & 1.359 & 1.365 & 1.37 \\
\hline 10 & 1.564 & 1.564 & 1.564 & 1.564 & 1.564 & 1.564 & 1.564 & 1.564 & 1.564 & 1.564 & \\
\hline
\end{tabular}
в начальный момент времени, $T_{i, \frac{m}{2}}$ - на середине, $T_{i, m}$ - распространение температуры в конце процесса.

Таблица 1. Результаты вычисления значений температуры по времени

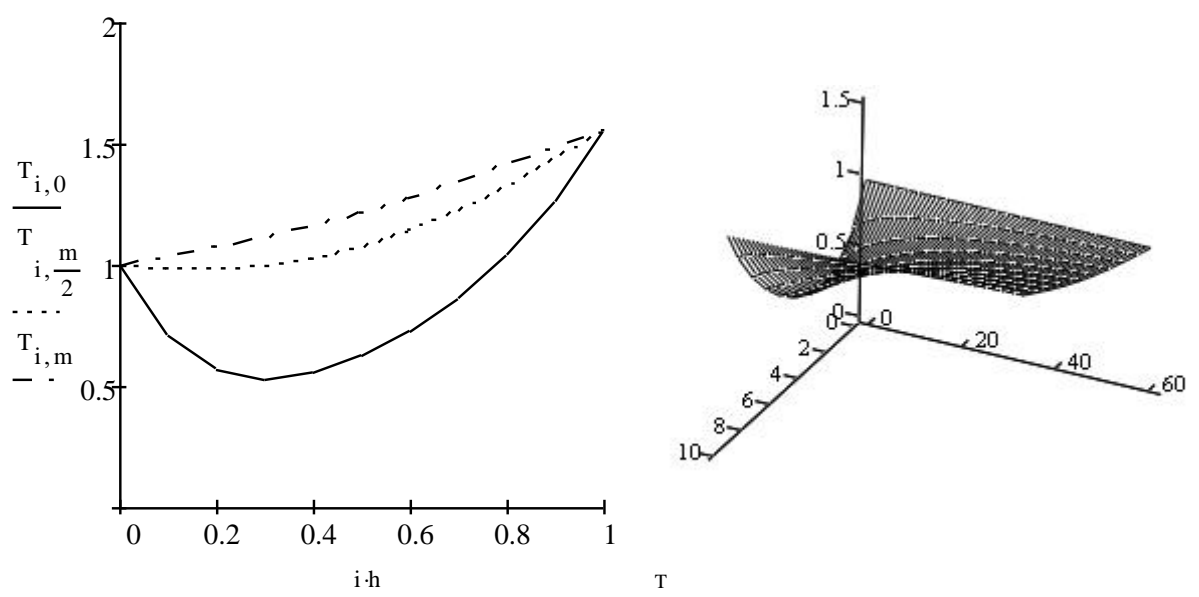

Рис. 1. Двумерный и трехмерный график распространения температуры при $0 \leq x \leq 1,0 \leq t \leq 0,25$

\section{Список литературы / References}

1. Калиткин Н.Н. Численные методы. М.:Наука,1978. 512 с.

2. Самарский А.А., Николаев Е.С. Методы решении сеточных уравнении. М.: Наука, 1978. 588 с. 